\title{
TOWARD A HISTORICAL SOCIOLINGUISTIC POETICS OF MEDIEVAL GREEK
}




\title{
BYZANTIOS \\ Studies in Byzantine History and Civilization
}

I 2

\author{
Series Editors \\ Michael Altripp \\ Lars Martin Hoffmann \\ Christos Stavrakos \\ Editorial \& Advisory Board \\ Michael Featherstone (CNRS, Paris) \\ Bojana Krsmanović (Serbian Academy of Sciences and Arts, Belgrade) \\ Bogdan Maleon (University of Iasi) \\ Antonio Rigo (University of Venice) \\ Horst Schneider (University of Munich) \\ Juan Signes Codoner (University of Valladolid) \\ Peter Van Deun (University of Leuven) \\ Nino Zchomelidse (Johns Hopkins University)
}




\section{TOWARD A HISTORICAL SOCIOLINGUISTIC POETICS OF MEDIEVAL GREEK}

Edited by

Andrea M. CuOmo \& Erich TrapP

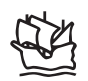

BREPOLS 
(C) 2017, Brepols Publishers n.v., Turnhout, Belgium.

All rights reserved. No part of this publication may be reproduced, stored in a retrieval system, or transmitted, in any form or by any means, electronic, mechanical, photocopying, recording, or otherwise without the prior permission of the publisher.

$\mathrm{D} / 2017 / 0095 / 236$

ISBN 978-2-503-577 I 3-5

E-ISBN 978-2-503-577 I 4-2

DOI I o.I 484/M.SBHC-EB.5. I I 3947

Printed on acid-free paper. 


\section{TABLE OF CONTENTS}

Vorwort

Andrea Massimo Cuomo, Historical Sociolinguistics - Pragmatics and Semiotics, and the Study of Medieval Greek Literature

Klaas Bentein, Towards a Socio-Historical Analysis of Ancient Greek? Some Problems and Prospects

Stefano Valente, Old and New Lexica in Palaeologan

Byzantium

Daniele Bianconi, La lettura dei testi antichi tra didattica ed erudizione: Qualche esempio d'età paleologa

Inmaculada PÉrez Martín, Aristides' Panathenaikos as a Byzantine Schoolbook: Nikephoros Gregoras' Notes on Ms. Escorial Ф.I. I 8

Geoffrey Horrocks, Georgios Akropolitis: Theory and Practice in the Language of Later Byzantine Historiography

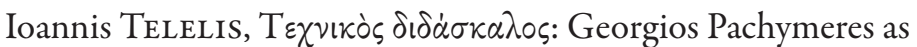
Paraphrast of Aristotelian Meteorology

Divna Manolova, The Student Becomes the Teacher: Nikephoros Gregoras' Hortatory Letter Concerning Astronomy

Paolo Odorico, Identité et craintes. Théodore Pédiasimos à Serrès au XIV siècle

Abstracts

Bibliography

General Index

Index of Manuscripts 



\section{VORWORT}

Der vorliegende Band enthält die Vorträge und Diskussionsbeiträge der internationalen Tagung vom September 2014 , zu der ein vorbereitender Workshop bereits im Juni 20 I 3 stattfand. Das Thema dieser Tagung entsprang dem vom FWF finanzierten Projekt 239 I 2-Gig "Imitation und Innovation im Wortschatz der spätbyzantinischen historiographischen Literatur".

Gleich zu Beginn unserer sprachlichen Untersuchungen der Werke der spätbyzantinischen Historiker stellte sich die Frage, welchen Weg wir beschreiten sollten: eher den herkömmlichen, positivistischen (Fragen des Stilniveaus, Metaphrasen, Übergang zur Volkssprache) oder einen neuen, der sich den Texten mit folgender Frage zu nähern sucht: Wie mag eine syntaktische Konstruktion, die Wahl eines besonderen Wortes usw. in den Ohren eines Byzantiners geklungen haben? Um diese Veränderung der Perspektive zu erreichen, galt es, die Methoden der historischen Soziolinguistik anzuwenden. Diese hat - insbesondere für das mittelalterliche Englisch - die sprachlichen Kategorien aufgezeigt, nach welchen die Sprecher ihre Sprache verstanden, beurteilten und mit ihr umgingen. Demnach sind die Texte möglichst ohne den Filter der neuzeitlichen und damit anachronistischen Kategorien zu interpretieren.

Der vorliegende Band enthält also Beiträge zur historischen Soziolinguistik und ihren Grenzen, ohne freilich bereits eine grundlegende Abhandlung über die griechische Sprache des Mittelalters darzustellen. Vielmehr wird der Leser unsere Auseinandersetzungen mit der methodologischen Problematik der historischen Soziolinguistik nachverfolgen können. Er wird entdecken, wie (und in welchem Ausmaß) der "ideale Leser" auch Mit-Autor eines Werkes wird. Er wird sich einnehmen lassen von den spannenden Schwierigkeiten, welche die Auffindung und Sammlung der Urteile der Byzantiner über ihre Sprache betreffen. Jene zeitgenössischen Beobachtungen erlauben uns beispielsweise zu verstehen, welche Wörter und welche syntaktischen Konstruktionen die Byzantiner als "Attisch" oder als "Koine" betrachteten, und welche pragmatische Funktion eine Varietät in ihrem jeweiligen Kontext innehatte. Historische Soziolinguistik bedeutet nicht nur, das Publikum und den Kontext zu berücksichtigen! Vielmehr fordert die historische Soziolinguistik uns dazu auf, zeitgenössische sprachliche Beobachtungen zu fin- 
den, zu sammeln und zu bewerten, um die soziale Funktion der Sprache und ihrer Varianten verstehen und rekonstruieren zu können.

Der Wert dieses Sammelbandes soll weniger in den erzielten Einzelbeobachtungen bestehen als in der verfolgten Methode, auch wenn es sich zunächst nur um erste Schritte auf einem Weg mit noch ungewissem Ziel handeln kann. Aber gerade deshalb möchten wir den Kollegen besonders danken, die unserer Einladung nach Wien gefolgt sind, an den sehr konstruktiven Diskussionen teilgenommen und schließlich durch die schriftliche Fassung ihrer Beiträge das Zustandekommen der vorliegenden Publikation ermöglicht haben.

Wien, August 2016

Die Herausgeber 